\title{
Femtosecond Laser Inscribed Y-Branch Waveguide in Nd:YAG Crystal: Fabrication and Continuous-Wave Lasing
}

\author{
Hongliang Liu, Javier R. Vázquez de Aldana, Minghui Hong, and Feng Chen
}

\begin{abstract}
Rectangular Y-branch cladding waveguides have been fabricated in Nd:YAG crystal by femtosecond laser inscription. Such novel configurations are fabricated with depth of $50 \mu \mathrm{m}$, supporting multimode guidance in both TM and TE polarizations. Continuous wave laser oscillations at wavelength of $1.06 \mu \mathrm{m}$ have been achieved under the optical pump at $808 \mathrm{~nm}$. The maximum output power is $0.2 \mathrm{~W}$ with a slope efficiency of $20 \%$ in the device with splitting angle of $0.5^{\circ}$.
\end{abstract}

Index Terms-Optical waveguides, femtosecond laser inscription, waveguide lasers, Y-branch waveguides.

\section{INTRODUCTION}

A S THE basic components in integrated optics, waveguides possess advantages of confinement of the light propagation compactly in small volumes, in which higher optical density could be achieved with respect to bulk materials, which is beneficial to the construction of high-performance photonic devices in small scales of dimensions [1], [2]. In active media, the compressed volume in waveguide geometries leads to high optical intensities of pump beams inside the structures, and results in laser oscillations in waveguides with reduced threshold and enhanced efficiency with respect to the bulks [3]-[5]. The femtosecond laser inscription (FLI) has been first reported to be utilized to fabricate optical waveguides in glasses in 1996 [6]. As an efficient technique for direct microprocessing of transparent dielectric materials, the FLI possesses unique 3-D capability for device production compared with other traditional waveguide fabrication techniques, such as ion exchange, ion

Manuscript received March 18, 2015; revised May 22, 2015; accepted May 26, 2015. Date of publication June 1, 2015; date of current version August 20, 2015. This work was supported by the National Natural Science Foundation of China (11274203), the Specialized Research Fund for the Doctoral Program of Higher Education of China (20130131130001) and Junta de Castilla y León under Project SA086A12-2, and Ministerio de Economía y Competitividad (under Project FIS2013-44174-P), Spain, and the Competitive Research Program of Singapore under CRP Award NRF-CRP10-2012-04.

H. Liu is with the School of Physics, State Key Laboratory of Crystal Materials, Shandong University, Jinan 250100, China, and also with the Department of Electrical and Computer Engineering, National University of Singapore, 117576 Singapore (e-mail: liuh18708@gmail.com)

J. R. Vázquez de Aldana is with the Laser Microprocessing Group, Facultad Ciencias, Universidad de Salamanca, Salamanca 37008, Spain (e-mail: jrval@usal.es).

M. Hong is with the Department of Electrical and Computer Engineering, National University of Singapore, 117576 Singapore (e-mail: elehmh@nus.edu.sg).

F. Chen is with the School of Physics, State Key Laboratory of Crystal Materials, Shandong University, Jinan 250100, China (e-mail: drfchen@ sdu.edu.cn).

Color versions of one or more of the figures in this paper are available online at http://ieeexplore.ieee.org.

Digital Object Identifier 10.1109/JSTQE.2015.2439191 irradiation [7]-[9]. Focused femtosecond-laser pulses produce localized modification in micro- or sub-micrometer scales in the bulk materials. The parameters of the femtosecond laser pulses, such as wavelength, pulse energy, pulse duration, polarization as scanning speed [3], [9], [10], are critical for the refractive index modifications of the materials. Waveguides fabricated by FLI have been realized in a number of gain crystals and ceramics, e.g., rare-earth ions ( $\mathrm{Nd}$ or $\mathrm{Yb}$ ) doped neodymium doped yttrium aluminum garnet (Nd: $\mathrm{Y}_{3} \mathrm{Al}_{5} \mathrm{O}_{12}$ or Nd:YAG) [11]-[15], GGG [16], KGW [17], vanadate crystals $\left(\mathrm{GdVO}_{4}\right.$ and $\left.\mathrm{YVO}_{4}\right)$ [18], [19] as well as nonlinear crystals (such as $\mathrm{BiBO}_{3}$ and $\mathrm{KTiOPO}_{4}$ ) [20], [21], and in various glasses [22]. The depressed cladding waveguides confine light propagation in channels which are surrounded by numbers of low-index tracks, different from dualline waveguides which are located in the region between two filaments with reduce indices [5], [23]. In addition, in cubic crystals of YAG or GGG, the dual-line structures only support light propagating at TM polarization, whilst the cladding structures enable mode confinement at any transverse polarizations.

Nd:YAG crystal is one of the most widely used gain media for solid-state lasers for its outstanding fluorescence, thermal and mechanical properties. Depressed cladding waveguide laser have been achieved in Nd:YAG crystal and ceramics [11]-[13]. Y-branch waveguide, which could act as an optical amplifier or splitter in the integrated optical devices, have already been fabricated in Nd-doped silicate glass, $\mathrm{LiNbO}_{3}$, BK-7 glass and Yb:YAG [24]-[27]. Meanwhile, the Y-branch construction could be used for monolithically integrated optical heterodyne systems with designed for signal amplification and communication applications [24], [28], [29]. Furthermore, the Y-branch splitter serve as the basic element to realize the wavelength division multiplexing in the optical networks [28].

In this work, we report on the fabrication of rectangular Ybranch surface cladding waveguides with different splitting angles by FLI in Nd:YAG crystal. For comparison, straightforward rectangular Y-branch cladding waveguides were produced in the surface of the bulk. Continuous wave (cw) lasing experiments were taken under the direct optical pump of the structures at $808 \mathrm{~nm}$.

\section{EXPERIMENTS IN DETAILS}

The Nd:YAG crystal sample (doped by 1 at. $\% \mathrm{Nd}^{3+}$ ions) was cut with sizes of $10 \times 10 \times 2 \mathrm{~mm}^{3}$. The Y-branch depressed cladding waveguides were produced by using the laser facility of the Universidad de Salamanca, Spain. A Ti:Sapphire 
TABLE I

CONSTANTS OF Y-BRANCH CLADDING WAVEGUIDES

\begin{tabular}{lccc}
\hline \hline No. & Width (single/branch sections) $(\mu \mathrm{m})$ & Depth $(\mu \mathrm{m})$ & Splitting angle $\left(^{\circ}\right)$ \\
\hline $\operatorname{Pr} 1$ & $50 / 25+25$ & 50 & 2 \\
$\operatorname{Pr} 2$ & $50 / 25+25$ & 50 & 1 \\
$\operatorname{Pr} 3$ & $50 / 25+25$ & 50 & 0.5 \\
$\operatorname{Pr} 4$ & 50 & 50 & 0 \\
\hline \hline
\end{tabular}

regenerative amplifier (Spitfire, Spectra Physics, USA), delivering linearly-polarized pulses of $120 \mathrm{fs}$ and $795 \mathrm{~nm}$ central wavelength at $1 \mathrm{kHz}$ repetition rate as previous report [12], [16]-[21], was used as laser source. The maximum available pulse energy was $1 \mathrm{~mJ}$. In order to get a fine control of the incident energy, it was reduced with a calibrated neutral density filter placed after a half-wave plate and a linear polarizer.

During the process of fabricating the Y-branch depressed cladding waveguides, the pulse energy was set to $15 \mu \mathrm{J}$ and the laser beam was focused with a $40 \times$ microscope objective (N.A. $\sim 0.6$ ) at a depth of $50 \mu \mathrm{m}$ beneath one of the $10 \times 10 \mathrm{~mm}^{2}$ surfaces. The sample was scanned at a constant velocity of $500 \mu \mathrm{m} / \mathrm{s}$ in the direction paralleled to the $10-\mathrm{mm}$ edge, producing a damage line along the sample. The procedure was repeated at different depths of the sample, following the desired rectangular geometry with a lateral separation of $3 \mu \mathrm{m}$ between each two adjacent tracks. Under these conditions, rectangular-shape structures were achieved, and the parameter of the novel structures could be found in Table I. The length of the single section and the branch section are 3.5 and $6.5 \mathrm{~mm}$, respectively. According to the micromachining parameters, the volume ration between the two branch sections is about 1:1. The splitting angle $\theta$ of the configurations $\operatorname{Pr} 1-\operatorname{Pr} 3$ was arranged from $0.5^{\circ}$ to $2^{\circ}$. For comparison, straightforward depressed cladding waveguide $(\operatorname{Pr} 4)$ was manufactured in the bulk materials under the same situation. Microscope images of the Y-branch cladding waveguides (input face, top surface and output face) are shown in Fig. 1(a)-(c).

With the measurement of the N.A. of the waveguides and the formula reported in [30], the maximum changes of refractive index of the waveguides was estimated to be $3.4 \times 10^{-3}$. In spite of the method itself being a rough estimation, this value was in good agreement with those of other cladding waveguides. Afterwards, the back-reflection method was used to measure the losses of the waveguides at wavelength of $632.8 \mathrm{~nm}$ with a $\mathrm{He}-\mathrm{Ne}$ laser as the light source at room temperature [31]. The propagation loss of the straightforward waveguide was about $1.1 \mathrm{~dB} / \mathrm{cm}$. The endface coupling experiments with a $1.06-\mu \mathrm{m}$ solid-state laser were performed and the output power of the Y-branch constructions $(\operatorname{Pr} 1$ - $\operatorname{Pr} 3)$ was nearly the same to that of the straightforward waveguide $\operatorname{Pr} 4$, which was mainly due to the similar guiding volume. Compared with the losses of the straightforward cladding structure, additional losses from the Y-branch splitting geometry is negligible, which means lossless splitting structures were produced by femtosecond laser writing in this work.

A wavelength-tunable cw Ti:Sapphire laser (Coherent MBR PE, USA) generating a polarized light pump beam at $808 \mathrm{~nm}$ was

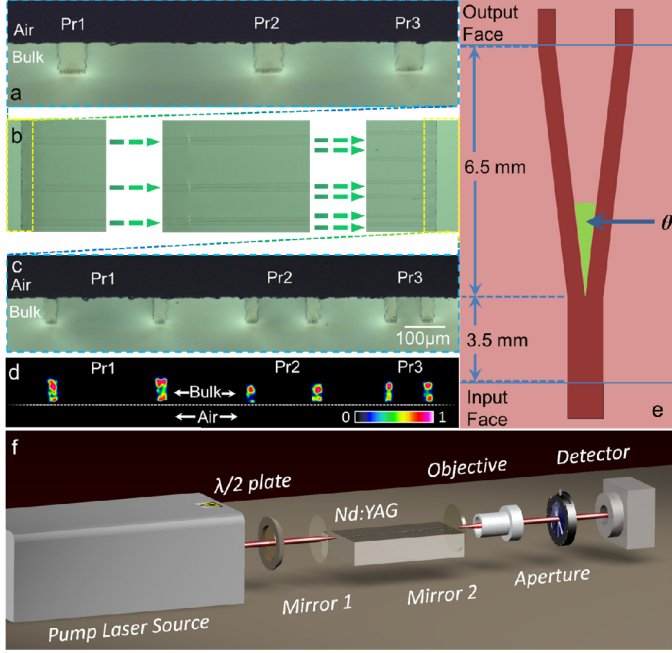

Fig. 1. Microscope images of the input face (a), top surface (b) and output face (c) of the Y-branch cladding waveguides in Nd:YAG crystal; (d) cw laser modal profiles at $1064 \mathrm{~nm}$ collected from the structures at TM polarization. (e) Schematic diagram of the Y-branch splitter configuration, $\theta$ is the splitting angle. (f) Schematic diagram of the experimental setup for the $\mathrm{cw}$ waveguide laser oscillation in the Y-branch cladding waveguides.

used in the end pumping system to performed the cw Y-branch waveguide laser-operation experiments as shown in Fig. 1(f). Spherical convex lens with a focal length of $25 \mathrm{~mm}$ was used to couple the pump laser beam into the guiding structures. A $20 \times$ long working distance microscope objective (N.A. $=0.4$ ) was used to collect the generated waveguide lasers and an IR CCD camera to image the laser beam profiles through an aperture. A spectrometer was used to analyze the emission spectra of the pump source and the generated laser beam.

\section{RESUlTS AND DISCUSSION}

During the endface coupling experiments for light transmissions at wavelength of $1.06 \mu \mathrm{m}$, modal profiles of output laser at TM polarization were measured. As shown in Fig. 1(d), the laser modal profiles are not at fundamental modes or single modes as reported previously [12]. This is mainly caused by the edge of the rectangle structures, which is not as smooth as the circular structures. The 1.06- $\mu \mathrm{m}$ light propagation in Y-branch waveguides was simulated by the well-known Rsoft software and shown in Fig. 2 [32]. The left, middle and right figures are corresponding to the Y-branch waveguides with splitting angles of $2^{\circ}, 1.5^{\circ}$ and $0.5^{\circ}$, respectively. The sources were set as Gaussian beams and the lengths of the waveguides were set to be $10 \mathrm{~mm}$ that are in good agreement with the structure fabricated by FLI. From the simulated images, disordered modes could be found which is also confirmed in the cw laser modal profiles displayed in Fig. 1(d).

Fig. 3 displays the spectrum of the pumping laser (dashed line) and output laser oscillation (solid line) when the pumping laser is above the threshold. The 808-nm laser with the full width at half maximum (FWHM) of $\sim 0.6 \mathrm{~nm}$ is corresponding to main absorption transition of $\mathrm{Nd}^{3+}$ ions, meanwhile the generated 1064-nm laser oscillation line with a FWHM of $0.4 \mathrm{~nm}$ 


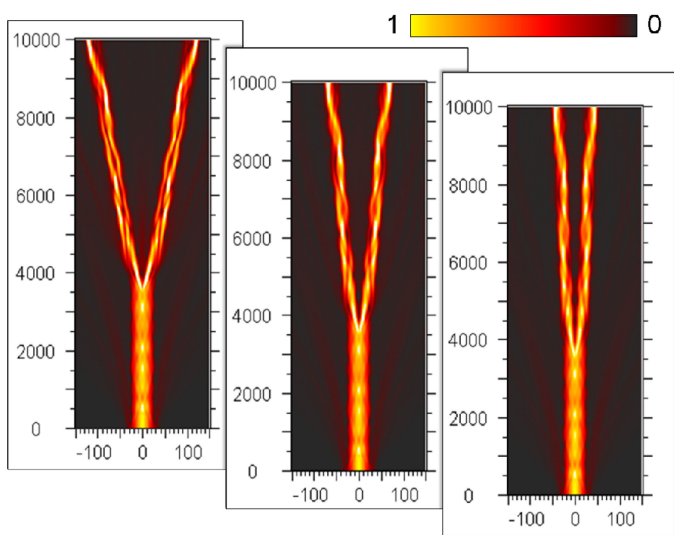

Fig. 2. Simulated the $1.06-\mu \mathrm{m}$ transformation status in Y-branch waveguides with different splitting angle $\theta$ arranging from $2^{\circ}$ to $0.5^{\circ}$.

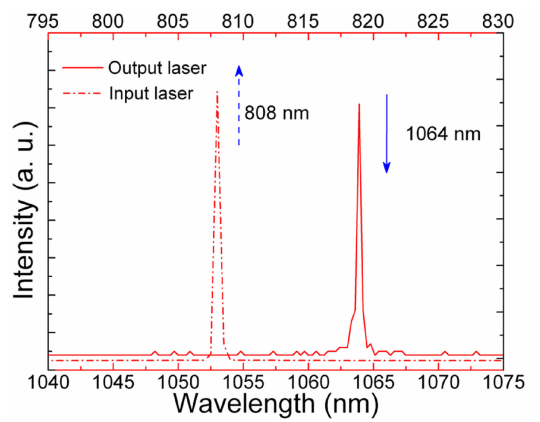

Fig. 3. Spectra of the pumping laser (dashed line) and output laser oscillation (solid line) when pumping laser is above the threshold at room temperature. FWHM of the lasers are 0.6 and $0.4 \mathrm{~nm}$, respectively.

is corresponding to the main fluorescence of ${ }^{4} \mathrm{~F}_{3 / 2} \rightarrow{ }^{4} \mathrm{I}_{11 / 2}$ transition of $\mathrm{Nd}^{3+}$ ions.

Waveguiding laser features of the Y-branch cladding structures were calculated with consideration of the transmittance and reflectivity of the optical elements (e.g., microscope lenses) in the end-coupling experiment system. As shown in Fig. 4(a), the linearly-polarized pumping laser was changed through adjusting half-wavelength plate, and the resultant all-angle performance of the output laser collected from the cladding waveguides proves that the laser oscillations in the rectangular cavity are not influenced by the polarizations of the pumping laser. The fitting lines show the maximum output power of the $1.06-\mu \mathrm{m}$ laser when the input laser power was about 1.1 W. Difference between the output powers of lasers collected from the cladding waveguides (Pr1 - Pr4) is mainly owing to the splitting angles of the configurations. Despite the losses measured in the endface coupling experiment at $1.06 \mu \mathrm{m}$ were nearly the same for the structures, the effect of the splitter junction caused in the laser oscillation could not be neglected. Fig. 4(b) displays the output powers of the active cw lasers versus the launched power at TM polarization. The slope efficiency of the output laser calculated $\left(\eta_{P r 1}, \eta_{P r 2}, \eta_{P r 3}\right.$ and $\left.\eta_{P r 4}\right)$ are $18.1 \%, 18.7 \%$, $20.2 \%$ and $22.4 \%$, respectively. Some other laser features of Y-branch rectangular cladding waveguides are depicted in Table II. The output power ratio between left and right branch are all about $49: 51$, which is mostly caused by the little dif-

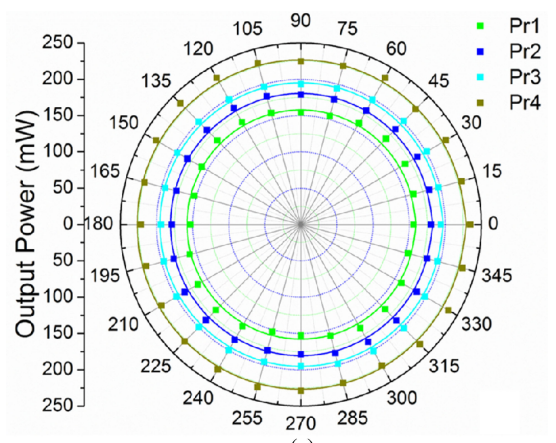

(a)

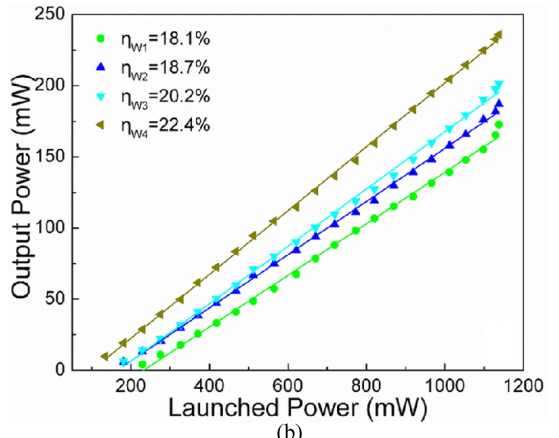

(b)

Fig. 4. (a) All-angle performance of the output power of the active laser collected from the Y-branch waveguides (Pr1 - Pr3) and the straightforward rectangular cladding waveguide (Pr4) when the launched power was $1.1 \mathrm{~W}$. The incident angle of the 808-nm laser pumping laser was changed from $0^{\circ}$ to $360^{\circ}$. (b) Output power of the collected laser as functions of the launched power. The slope efficiency of the output power were obtained after calculation.

TABLE II

LASER PERFormance of Y-BRANCH CladDing WAVEGUides

\begin{tabular}{|c|c|c|c|c|}
\hline No. & $\begin{array}{l}\text { Output power ratio } \\
\text { between left and right } \\
\text { branch }(1064 \mathrm{~nm})\end{array}$ & & $\begin{array}{c}\text { Ratio of Output } \\
\text { power compared with } \\
\text { that of } \operatorname{Pr} 4\end{array}$ & $\begin{array}{c}\text { Maximum output } \\
\text { power } \\
(\mathrm{mW})\end{array}$ \\
\hline Pr1 & $49.2: 50.8$ & \multirow{4}{*}{ Approximately 49:51 } & $81.5: 100$ & 172 \\
\hline $\operatorname{Pr} 2$ & $48.7: 51.3$ & & $87: 100$ & 187 \\
\hline $\operatorname{Pr} 3$ & $48.8: 51.2$ & & $92.3: 100$ & 201 \\
\hline $\operatorname{Pr} 4$ & & & $100: 100$ & 236 \\
\hline
\end{tabular}

ference of the laser cavity volume. With the splitting angle increases from $0^{\circ}$ to $2^{\circ}$, the maximum output laser power decreases from 236 to $172 \mathrm{~mW}$. Meanwhile, threshold of the laser oscillation are $99,166,164$ and $231 \mathrm{~mW}$, respectively. The maximum output power of the Y-branch cladding waveguide with splitting angle of $0.5^{\circ}$ is $92.3 \%$ of the straightforward cladding waveguides, which demonstrates the excellent performance of Y-branch splitter structure fabricated in Nd:YAG crystal by FLI.

\section{CONCLUSION}

We have demonstrated the fabrication of depressed cladding Y-branch waveguides in Nd:YAG crystals by the FLI. Rectangular Y-branch cladding waveguides with different splitting angles $\left(0^{\circ}, 0.5^{\circ}, 1^{\circ}\right.$ and $\left.2^{\circ}\right)$ were manufactured in the cubic Nd:YAG crystal with acceptable guiding properties. Under direct optical pump at $808 \mathrm{~nm}$, the cw laser operating at $1064 \mathrm{~nm}$ with a maximum output powers of up to $201 \mathrm{~mW}$ has been obtained in the Nd:YAG Y-branch waveguide laser system with a splitting angle 
of $0.5^{\circ}$. Excellent performances of the novel Y-branch waveguiding systems confirm that such promising devices could be integrated in optical devices for diverse applications.

\section{REFERENCES}

[1] E. J. Murphy, Intergrated Optical Circuits and Components. New York, NY, USA: Marcel Dekker, 1999.

[2] C. Grivas, "Optically pumped planar waveguide lasers, part I: Fundamental and fabrication techniques," Prog. Quantum Electron., vol. 35, no. 6, pp. 159-239, 2011.

[3] F. Chen and J. R. Vázquez de Aldana, "Optical waveguides in crystalline dielectric materials produced by femtosecond-laser micromachining," Laser Photon. Rev., vol. 8, no. 2, pp. 251-275, 2014.

[4] M. Ams, G. D. Marshall, P. Dekker, J. Piper, and M. Withford, "Ultrafast laser written active devices," Laser Photon. Rev., vol. 3, no. 6, pp. 535-544, 2009.

[5] Y. Tan et al., "70\% slope efficiency from an ultrafast laser-written $\mathrm{Nd}: \mathrm{GdVO}_{4}$ channel waveguide laser," Opt. Exp., vol. 18, no. 24, pp. 24994-24999, 2010.

[6] K. M. Davis, K. Miura, N. Sugimoto, and K. Hirao, "Writing waveguides in glass with a femtosecond laser," Opt. Lett., vol. 21, no. 21, pp. 17291731,1996

[7] R. R. Gattass and E. Mazur, "Femtosecond laser micromachining in transparent materials," Nat. Photon., vol. 2, no. 4, pp. 219-225, 2008.

[8] Y. Jia, N. Dong, F. Chen, J. R. Vázquez de Aldana, S. Akhmadaliev, and S. Zhou, "Ridge waveguide laser in Nd:GGG crystals produced by swift carbon ion irradiation and femtosecond laser ablation," Opt. Exp., vol. 20, no. 9, pp. 9763-9768, 2012

[9] S. Nolte, M. Will, J. Burghoff, and A. Tünnermann, "Femtosecond waveguide writing: A new avenue to three-dimensional integrated optics," Appl. Phys. A, vol. 77, no. 1, pp. 109-111, 2003.

[10] J. Burghoff, H. Hartung, S. Nolte, and A. Tunnermann, "Structural properties of femtosecond laser-induced modifications in $\mathrm{LiNbO}_{3}$," Appl. Phys., A Mater. Sci. Process., vol. 86, no. 2, pp. 165-170, 2006.

[11] G. Salamu, F. Jipa, M. Zamfirescu, and N. Pavel, "Laser emission from diode-pumped Nd:YAG ceramic waveguide lasers realized by direct femtosecond-laser writing technique," Opt. Exp., vol. 22, no. 5, pp. 5177 $5182,2014$.

[12] H. Liu, F. Chen, J. R. Vázquez de Aldana, and D. Jaque, "Femtosecond laser inscribed double cladding waveguides in Nd:YAG crystal: A promising prototype for integrated lasers," Opt. Lett., vol. 38, no. 17, pp. 3294-3297, 2013.

[13] N. Pavel et al., "Efficient laser emission in diode-pumped Nd:YAG buried waveguides realized by direct femtosecond-laser writing," Laser Phys. Lett., vol. 10, no. 9, art. no. 095802, 2013.

[14] G. A. Torchia et al., "Highly efficient laser action in femtosecond-written Nd:yttrium aluminum garnet ceramic waveguides," App. Phys. Lett., vol. 92 , no. 11, art. no. $111103,2008$.

[15] J. Siebenmorgen, T. Calmano, K. Petermann, and G. Huber, "Highly efficient $\mathrm{Yb}$ :YAG channel waveguide laser written with a femtosecondlaser," Opt. Exp., vol. 18, no. 15, pp. 16035-16041, 2010.

[16] C. Zhang et al., "Channel waveguide lasers in Nd:GGG crystals fabricated by femtosecond laser inscription," Opt. Exp., vol. 19, no. 13, pp. 1250312508, 2011.

[17] H. Liu, Q. An, F. Chen, J. R. Vázquez de Aldana, and B. del Rosal Rabes, "Continuous-wave lasing at $1.06 \mu \mathrm{m}$ in femtosecond laser written Nd:KGW waveguides," Opt. Mater., vol. 37, pp. 93-96, 2014.

[18] H. Liu, Y. Tan, J. R. Vázquez de Aldana, and F. Chen, "Efficient laser emission from cladding waveguide inscribed in $\mathrm{Nd}: \mathrm{GdVO}_{4}$ crystal by direct femtosecond laser writing," Opt. Lett., vol. 39, no. 15, pp. 45534556, 2014.

[19] Y. Jia, F. Chen, and J. R. Vázquez de Aldana, "Efficient continuous-wave laser operation at $1064 \mathrm{~nm}$ in $\mathrm{Nd}: \mathrm{YVO}_{4}$ cladding waveguides produced by femtosecond laser inscription," Opt. Exp., vol. 20, no. 15, pp. 1680116806, 2012.

[20] Y. Jia, J. R. Vázquez de Aldana, Q. Lu, D. Jaque, and F. Chen, "Second harmonic generation of violet light in femtosecond-laser-inscribed $\mathrm{BiB}_{3} \mathrm{O}_{6}$ cladding waveguides," Opt. Mater. Exp., vol. 3, no. 9, pp. 1279-1284, 2013.

[21] N. Dong, F. Chen, and J. R. Vázquez de Aldana, "Efficient second harmonic generation by birefringent phase matching in femtosecond-laserinscribed KTP cladding waveguides," Phys. Status Solidi RRL, vol. 6, no. 7, pp. 306-308, 2012
[22] M. Ams, P. Dekker, G. D. Marshall, and M. J. Withford, "Monolithic $100 \mathrm{~mW} \mathrm{Yb}$ waveguide laser fabricated using the femtosecond-laser direct-write technique," Opt. Lett., vol. 34, no. 3, pp. 247-249, 2009.

[23] R. He, I. Hernández-Palmero, C. Romero, J. R. Vázquez de Aldana, and F. Chen, "Three-dimensional dielectric crystalline waveguide beam splitters in mid-infrared band by direct femtosecond laser writing," Opt. Exp., vol. 22, no. 25, pp. 31293-31298, 2014.

[24] N. A. Sanford, K. J. Malone, D. R. Larson, and R. K. Hickernell, "Ybranch waveguide glass laser and amplifier," Opt. Lett., vol. 16, no. 15, pp. 1168-1170, 1991.

[25] E. K. Mwarania, D. M. Murphy, M. Hempstead, L. Reekie, and J. S. Wilkinson, "Integrated Q-switched multiple-cavity glass waveguide laser," IEEE Photon. Technol. Lett., vol. 4, no. 3, pp. 235-237, Mar. 1992.

[26] N. A. Sanford, J. A. Aust, K. J. Malone, and D. R. Larson, "Linewidth narrowing in an imbalanced Y-branch waveguide laser," Opt. Lett., vol. 18, no. 4, pp. 281-283, 1993.

[27] T. Calmano, C. Kränkel, and G. Huber, "Laser oscillation in Yb:YAG waveguide beam splitter with variable splitting ratio," Opt. Lett., vol. 40, no. 8, pp. 1753-1756, 2015.

[28] J. C. Cartledge, "Performance of $10 \mathrm{~Gb} / \mathrm{s}$ lightwave systems based on lithium niobate Mach-Zehnder modulators with asymmetric Y-branch waveguides," IEEE Photon. Technol. Lett., vol. 7, no. 9, pp. 1090-1092, Sep. 1995.

[29] Y. Jia et al., "Monolithic crystalline cladding microstructures for efficient light guiding and beam manipulation in passive and active regimes," Sci. Rep., vol. 4, art. no. 5988, 2014.

[30] J. Siebenmorgen et al., "Femtosecond laser written stress-induced Nd: $\mathrm{Y}_{3} \mathrm{Al}_{5} \mathrm{O}_{12}$ (Nd:YAG) channel waveguide laser," Appl. Phys. B, vol. 97, no. 2, pp. 251-255, 2009.

[31] R. Ramponi, R. Osellame, and M. Marangoni, "Two straightforward methods for the measurement of optical losses in planar waveguides," Rev. Sci. Instrum., vol. 73, no. 3, pp. 1117-1120, 2002.

[32] Rsoft Design Group, computer software BeamPROP version 8.0 (2007). [Online]. Available: http://www.rsoftdesign.com

Hongliang Liu received the B.A. degree from Shandong University, Jinan, China, in 2011, where he is currently working toward the Ph.D. degree. His current research interests include fabrication of optical waveguides in laser materials by using ion beam implantation and femtosecond laser inscription technique.

Javier R. Vàzquez de Aldana received the B.S. and Ph.D. degrees from the University of Salamanca, Salamanca, Spain, in 1997 and 2001, respectively. $\mathrm{He}$ is currently an Associate Professor at the Science Faculty, University of Salamanca. His research interests include the interaction of intense femtosecond pulses with materials and its application to the fabrication of photonic devices. $\mathrm{He}$ is a Member of the Laser Microprocessing Research Group, and is also Technical and Scientific Advisor of the Laser Facility at the University of Salamanca.

Minghui Hong received the B.S. and M.S. degrees from Xiamen University, Xiamen, China, in 1985 and 1988, respectively. After teaching at Xiamen University from 1988 to 1994 , he continued his studies and received the M.E. and $\mathrm{Ph} . \mathrm{D}$. degrees from the National University of Singapore, Singapore, in 1996 and 2000, respectively. He is a currently a Research Scientist and a Professor at the Electrical and Computer Engineering Department, National University of Singapore. His research interests include laser ablation and its applications in microprocessing and nanofabrication. He is a Fellow of the Optical Society of America and the International Society of Optics and Photonics.

Feng Chen received the Ph.D. degree from Shandong University, Shandong, China, in 2002. He was with the Clausthal University of Technology, Germany, from 2003 to 2005, as an Alexander von Humboldt Research Fellow. He has been a Professor at Shandong University since 2006, and is also the Head of the School of Physics, Shandong University. His research interests include material modifications by ultrafast lasers and ion beams, optical waveguides, etc. He is a Fellow of the Institute of Physics, U.K., a Senior Member of the Optical Society of America and Chinese Optical Society. He also serves as an Associate Editor of Optical Engineering. 